\title{
TEM Waves Guided between Many Conductors
}

\author{
Reuven Ianconescu ${ }^{1}$ \\ ${ }^{1}$ Shenkar College of Engineering and Design, Ramat Gan, Israel \\ Correspondence: Reuven Ianconescu, Shenkar College of Engineering and Design, Ramat Gan, Israel. E-mail: \\ riancon@mail.shenkar.ac.il, riancon@gmail.com
}

Received: August 16, 2013 Accepted: October 16, 2013 Online Published: November 28, 2013

doi:10.5539/mas.v7n12p90 URL: http://dx.doi.org/10.5539/mas.v7n12p90

\begin{abstract}
Guided TEM waves have been extensively investigated between 2 conductors. The multiconductor case, in spite of some important publications in this direction, did not get into the mainstream of the electromagnetic education, and therefore deserves some more attention. The simple case of lossless multiconductor TEM waves in homogeneous media is a good approximation for many practical cases and is easily derivable by usage of potentials only. In this work we derive the formalism for lossless multiconductor TEM waves in homogeneous media, and show several examples for the usage of this formalism.
\end{abstract}

Keywords: TEM, guided waves, crosstalk

\section{Introduction}

The purpose of this work is to show a simple derivation for TEM waves guided between many perfect conductors, in a homogeneous media and to develop multiconductor models and applications for several geometries.

Multiconductor transmission lines have been first analyzed in the orientation of power systems. Matrix methods for power systems trace back to the seminal works of Park $(1929,1933)$, and known as Park transformations. Methods similar to the above, known as DQO transformations and $\alpha \beta \gamma$ transformations have been developed to ease manipulations of 3 phase networks.

Traveling waves phenomena in polyphase systems have been analyzed by Wedepohl (1963) and Galloway (1964). Those works generalized the telegraph equations for more than one dimension, and introduced the term of characteristic self impedance and characteristic mutual impedance between phases. The orientation of those works was for polyphase power systems.

In 1988 Clayton R. Paul issued a book (Paul, 1994) on multiconductor transmission lines. This book treats from a very high theoretical level this subject, analyzing both homogeneous and non homogeneous media, the lossy and lossless case. In 1999, F. Olyslager issued a book (Olyslager, 1999) on transmission lines representation of waveguides. The waveguide modes are represented by $N$ dimensional transmission lines techniques - see also Olyslager et al. (1994).

However, this subject did not get yet into the main stream of electromagnetic education, and simple derivations for lossless multiconductor TEM are not available. Most practical cases of multiconductor transmission line may be approximated as TEM in the homogeneous model, the inhomogeneity being expressed by an average equivalent dielectric constant. The waves propagate in quasi TEM, which may be well approximated by TEM (Pozar, 2009). Also losses, if they are small, may be introduced a posteriori.

It appears that the homogeneous lossless case can be very easily derived and formulated using only the scalar and vector potentials, and we present this formulation in the current work. Some preliminary work has been presented at Ianconescu (2012).

In section 2 we define the general configuration which consists of any number of parallel perfect conductors in a homogeneous lossless medium, and show a summary of the basic formulation of guided TEM waves and their propagation characteristics. In section 3 we formulate the interface connections between source, termination and the multiconductor TEM transmission line, with some examples. In section 4 we formulate an alternative representation for the multiconductor guided TEM, with an example and in section 5 we perform a flat cable 
analysis for which we discuss the cross talk issue. In section 6 we generalize the results of section 2 for the time harmonic case, discuss the VSWR issue, and present a short example. The work is ended with some concluding remarks.

\section{Basics of Guided TEM}

We consider an arbitrary number $N+1$ of parallel perfect conductors in the $z$ direction. The cross section of each conductor is fixed, hence $z$ independent. The medium between the conductors is homogeneous having constant electric permittivity $\epsilon$ and magnetic permeability $\mu$. The general configuration is shown in Figure 1. The conductors are numbered $1,2, . . N, N+1$, where the "last" $(N+1)$ conductor is defined as common.

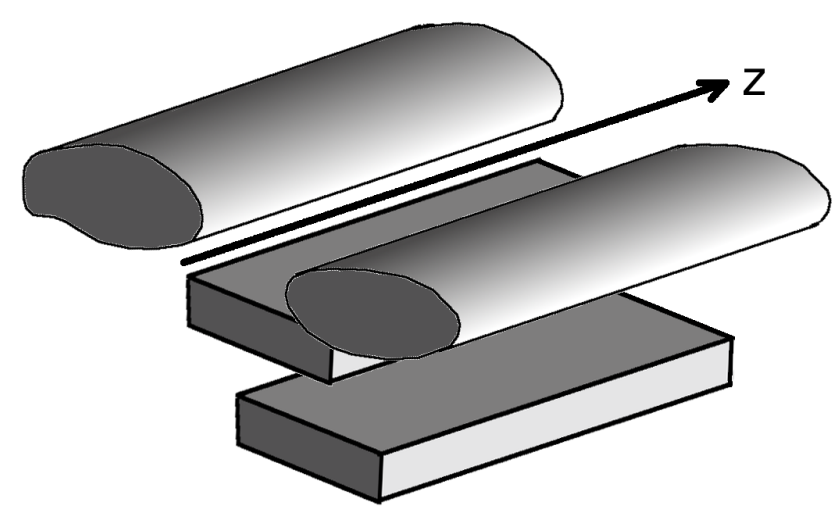

Figure 1. General configuration: several parallel perfect conductors (here shown 4 conductors), having a fixed cross section in the $x-y$ plane in a homogeneous lossless medium

A full electromagnetic derivation of the solution for such configuration can be found in the literature (Paul, 1994; Olyslager, 1999), although simpler derivations can be worked out for lossless TEM. Because the main results are known we present in the current section a summary of the most important results for perfect conductors in lossless homogeneous medium and the guidelines to derive them.

The scalar potential $V$, each component of the vector potential $\mathbf{A}$ and of the electric and magnetic fields, satisfy the homogeneous wave equation (Staelin, Morgenthaler, \& Kong, 1994; Pozar, 2009; Orfanidis, 2002; Chen \& Lu, 2011).

$$
\left(\nabla^{2}-\frac{1}{v^{2}} \partial_{t}^{2}\right) U=0
$$

where $U$ denotes any of the above entities, and $v \equiv \frac{1}{\sqrt{\epsilon \mu}}$ is the light velocity in the medium. The Lorentz gauge

$$
\nabla \cdot \mathbf{A}+\frac{1}{v^{2}} \partial_{t} V=0
$$

must also be satisfied. Now separating $\nabla^{2} \equiv \nabla_{\mathrm{tr}}^{2}+\partial_{z}^{2}$ in Equation (1), where $\nabla_{\mathrm{tr}}^{2}$ is the transversal Laplacian operator, we obtain

$$
\left(\nabla_{\mathrm{tr}}^{2}+\partial_{z}^{2}-\frac{1}{v^{2}} \partial_{t}^{2}\right) U=0
$$

By separating variables $U=U_{\text {tr }}(x, y) U_{l}(z, t)$ in Equation (3) one obtains

$$
\frac{\nabla_{\mathrm{tr}}^{2} U_{\mathrm{tr}}}{U_{\mathrm{tr}}}+\frac{\partial_{z}^{2} U_{l}-\frac{1}{v^{2}} \partial_{t}^{2} U_{l}}{U_{l}}=0,
$$

and the TEM are special solutions for which each part of Equation (4) is separately 0, namely

$$
\nabla_{\mathrm{tr}}^{2} U_{\mathrm{tr}}=0
$$

and

$$
\partial_{z}^{2} U_{l}-\frac{1}{v^{2}} \partial_{t}^{2} U_{l}=0
$$


Those special solutions exist only when the number of conductors is bigger than 1 (see Figure 1), because for a single conductor the Laplace Equation (5) solved for the scalar potential $V$, yields a constant solution, hence 0 electric field.

For our general configuration in Figure 1, the surface currents cannot be circular, because this implies non zero B field inside the conductors, which is impossible for perfect conductors. Hence the surface currents must be only in the $\widehat{z}$ direction, implying the vector potential to be $\mathbf{A}=A_{z} \widehat{z}$. So setting $V(x, y, z, t)=V_{\operatorname{tr}}(x, y) V_{l}(z, t)$ and $A_{z}(x, y, z, t)=A_{z \operatorname{tr}}(x, y) A_{z l}(z, t)$ and solving the one dimensional wave equation Equation (6) for $V_{l}(z, t)$ and $A_{z l}(z, t)$ yields any functions of a single variable, say $p$ which equals to $p=t \mp z / v$ :

$$
V_{l}(z, t)=f(p)=f(t \mp z / v)
$$

and

$$
A_{z l}(z, t)=g(p)=g(t \mp z / v)
$$

where $f$ and $g$ are any functions of a single argument, the upper and lower signs describing forward and backward moving waves, respectively.

To simplify, we shall go on for now only with the forward moving wave, because the backward moving wave describes the same physics in the opposite direction and one easily derives the backward solution from the forward solution. So we drop for now the lower sign.

Requiring now the Lorentz condition in Equation (2), we obtain

$$
\frac{v A_{z \operatorname{tr}}}{V_{\operatorname{tr}}}=\frac{f^{\prime}(t-z / v)}{g^{\prime}(t-z / v)} \equiv \kappa,
$$

where $f^{\prime}$ and $g^{\prime}$ are the derivatives of the functions $f$ and $g$ with respect to their argument. Now because the left side is only a function of $x, y$ and the right side is only a function of $z, t$, each must equal a constant which we name $\kappa$. So the right side yields $f^{\prime}(q)=\kappa g^{\prime}(q)$, where $q=t-z / v$ is the argument. We look for the dynamic solution for transients so by integrating from $q=-\infty$ to $q=p$, and assuming that $f(-\infty)=g(-\infty)=0$, i.e. no constant values before the transient started, we may express the solution for transients as $f(p)=\kappa g(p)$, namely:

$$
f(t-z / v)=\kappa g(t-z / v)
$$

Now combining the left side of Equation (9) with Equation (10) we find the connection between the general solution of $A_{z}$ and $V$

$$
A_{z}=A_{z \operatorname{tr}} g(t-z / v)=\frac{\kappa V_{\mathrm{tr}}}{v} \frac{f(t-z / v)}{\kappa}=\frac{V}{v},
$$

because $V=V_{\text {tr }} f(t-z / v)$. So we see that $\kappa$ cancels out and we obtain that at any coordinate $x, y, z$ and at any time $t$ the scalar potential $V$ equals to $v A_{z}$. So without loss of generality we may omit $\kappa$ by setting it to 1 , and use

$$
A_{z \mathrm{tr}}=\frac{V_{\mathrm{tr}}}{v} .
$$

By using the normal derivative boundary condition on $V_{\mathrm{tr}}$ and $A_{z \text { tr }}$ one derives from Equation (12) a connection between the surface charges and surface currents on the conductors, from which one derives the following connection:

$$
\mathbf{I}=v \boldsymbol{\lambda},
$$

where $\mathbf{I}$ and $\lambda$ are column vectors of size $N$, their components being the currents and and the charges per unit of length in the conductors, respectively.

The $N \times N$ elastance per unit of length matrix $\mathcal{P}$ is defined by the connection

$$
\mathbf{V}=\mathcal{P} \lambda
$$

where $\mathbf{V}$ is a column vector of size $N$, its components the voltages $V_{\text {tr }}$ on the conductors.

Now combining Equations (14) and (13), we obtain

$$
\mathbf{V}=\mathcal{Z}_{0} \mathbf{I}
$$


defining the characteristic impedance matrix

$$
\mathcal{Z}_{0} \equiv \mathcal{P} / v
$$

This is in analogy with the one dimensional case, i.e. $N+1=2$, for which the characteristic impedance is $Z_{0}=\frac{1}{v C^{\prime}}$, where $C^{\prime}$ is the capacity per unit of length.

We may as well define the vector $\mathbf{A}_{\mathbf{z}}$, its components being the magnetic potential vector $A_{z \text { tr }}$ on the conductors, and according to Equation (12), we have

$$
\mathbf{A}_{\mathbf{z}}=\frac{\mathbf{V}}{v}
$$

We now define the inductance matrix per length unit $\mathcal{L}$ by the connection

$$
\mathbf{A}_{\mathbf{z}}=\mathcal{L} \mathbf{I},
$$

and using Equations (17), (13) and (14), we obtain the connection $\mathcal{L}=\mathcal{P} / v^{2}$ or by defining the capacitance per unit length matrix $C=\mathcal{P}^{-1}$ and inserting the unit matrix $\mathcal{I}$, we may express

$$
\mathbf{C} \mathcal{L}=\mathcal{I} / v^{2}=\mathcal{I} \mu \epsilon
$$

which is the analogue of the formula $C^{\prime} L^{\prime}=\mu \epsilon$ for the one dimensional case. We may also express $\mathcal{Z}_{0}$ from Equation (16) as

$$
\mathcal{Z}_{0}^{2}=\mathcal{P}^{2} / v^{2}=\mathcal{L} \mathcal{P}=\mathcal{L} C^{-1},
$$

analogue to the formula $Z_{0}^{2}=L^{\prime} / C^{\prime}$ for the one dimensional case.

We shall calculate now the power carried by a forward moving wave, using Poynting theorem.

We first define $U_{E}^{\prime}$ as the electric energy per unit of length, which is calculated as half the sum on all conductors of the charge per length unit multiplied by the voltage, which may be put in matrix form as follows

$$
U_{E}^{\prime}=\frac{1}{2} \mathbf{V}^{T} C \mathbf{V}
$$

Similarly, we define $U_{H}^{\prime}$ as the magnetic energy per unit of length. It may be expressed as half the sum on $N$ conductors of the current multiplied by the magnetic vector potential and by using Equation (18), we express it as

$$
U_{H}^{\prime}=\frac{1}{2} \mathbf{I}^{T} \mathcal{L} \mathbf{I}
$$

For a forward moving wave, Equation (15) is satisfied, so by using it and the Equations (19) and (20), one easily shows that the electric and magnetic energies per length unit are equal, so that the total energy per length unit $U^{\prime}$ is given by

$$
U^{\prime}=U_{E}^{\prime}+U_{H}^{\prime}=2 U_{E}^{\prime}=\mathbf{V}^{T} C \mathbf{V}=\frac{1}{v} \mathbf{V}^{T} \mathcal{Z}_{0}^{-1} \mathbf{V} .
$$

We now apply the Poynting theorem at time $t$, on a volume enclosed between the surfaces $z=z_{1}$ and $z=z_{2}$

$$
P\left(t, z_{1}\right)-P\left(t, z_{2}\right)=\left.\frac{\partial}{\partial t} U\right|_{\text {inside volume }}=\frac{\partial}{\partial t} \int_{z_{1}}^{z_{2}} U^{\prime}(t, z) d z
$$

where $P$ is the forward power. We remember that any forward wave solution is not a function of the 2 separate variables $t$ and $z$, but rather a function of one variable $p=t-z / v$ (see Equations (7) and (8)), so that we may call

$$
U^{\prime}(t, z) \equiv h(p)=h(t-v / z),
$$

$h$ being the adequate function, according to the excitation of the system. So we obtain

$$
P\left(t, z_{1}\right)-P\left(t, z_{2}\right)=\int_{z_{1}}^{z_{2}} \frac{\partial}{\partial t} h(t-z / v) d z=\int_{z_{1}}^{z_{2}} h^{\prime}(t-z / v) d z,
$$

$h^{\prime}$ being the derivative of $h$ with respect to its argument $p$. We are left with a total derivative of a function having the argument linear in $z$, so integrating we obtain

$$
P\left(t, z_{1}\right)-P\left(t, z_{2}\right)=\left.\frac{h(t-z / v)}{-1 / v}\right|_{z_{1}} ^{z_{2}}=v\left[h\left(t-z_{1} / v\right)-h\left(t-z_{2} / v\right)\right] .
$$


Now we may take $z_{2} \rightarrow \infty$, so that the power and the function $h$ (which represents the energy per length unit) are 0 at $z=z_{2}$. So using Equations (23) and (25), we rewrite Equation (27) for $z_{1}$ being any $z$, knowing that everything is a function of $t-z / v$ :

$$
P(t-z / v)=\mathbf{V}^{T}(t-z / v) \mathcal{Z}_{0}^{-1} \mathbf{V}(t-z / v)
$$

which looks like a natural generalization of the forward power $P(t-z / v)=V^{2}(t-z / v) / Z_{0}$ for the one dimensional case.

So TEM waves guided by many conductors, may be specified in terms of voltages and currents, like in the two conductor case, but instead of the characteristic impedance we deal here with a characteristic impedance matrix, the voltages and currents being vectors.

\section{Termination and Source}

In this section we deal with the interface of the multiconductor transmission line with the load and with the feeding source.

In principle, one may treat the multiconductor transmission line as a $2 N$ port network, $N$ ports at feed, and $N$ ports at termination, see Paul (1994) Chapter 7.5, however this is usually an overcomplication.

The calculations are done for general excitation, and we consider for now only resistive loads and resistive source internal impedances.

\section{A. The Matching Network}

The matching network, i.e. the termination network of resistors that ensures zero reflected wave must satisfy the connection between voltages and currents dictated by the $\mathcal{Z}_{0}$ matrix. In other words, the matching network must have an impedance matrix $\mathcal{Z}$ that is identical to $\mathcal{Z}_{0}$.

The most general resistors network consists of one resistor between each pair of conductors. So starting to connect the resistors between conductor 1 and the other $N$ conductors, we have to connect $N$ resistors. Now, between conductor 2 and the others we have to connect only $N-1$ resistors, because between 1 and 2 we already connected, and so on, the total number of resistors is $N+(N-1)+. .+1=N(N+1) / 2$.

Let us call the resistor between conductor $i$ and conductor $N+1$ (which is the common), $R_{i}$, and let us call $R_{i j}$ the resistor between conductor $i$ and conductor $j$, where $i$ and $j$ have values between 1 and $N$. The resistors network has $N$ ports, port $i$ being defined between conductor $i$ and the common. We need to define a generic connection between those resistors and the impedance matrix of the network $\mathcal{Z}$.

From the definition of the impedance matrix $\mathcal{Z}$, the $j i$ component is

$$
Z_{j i}=\left.\frac{V_{j}}{I_{i}}\right|_{\substack{I_{k}=0 \\ k \neq i}} .
$$

The admittance matrix $\mathcal{Y}$ is the inverse of the impedance matrix, hence

$$
\mathcal{Y Z}=\mathcal{I}
$$

where $\mathcal{I}$ is the unit matrix. Equation (30) may be written in form of a sum, as follows:

$$
\sum_{k=1}^{N} Y_{m k} Z_{k i}=\delta_{m i}
$$

where $\delta$ is the Kronecker delta.

Now let us feed the network from port $i$ with a current $I_{i}$, leaving the other ports open (i.e. $I_{k}=0$ for $k \neq i$ ). The voltages at the ports are called $V_{j}$, for $j$ between 1 and $N$.

The KCL for port $i$ yields:

$$
I_{i}=\frac{V_{i}}{R_{i}}+\sum_{\substack{k=1 \\ k \neq i}}^{N} \frac{V_{i}-V_{k}}{R_{i k}},
$$


and the KCL for any other port $m$, where $m \neq i$, yields:

$$
0=\frac{V_{m}}{R_{m}}+\sum_{\substack{k=1 \\ k \neq m}}^{N} \frac{V_{m}-V_{k}}{R_{m k}} .
$$

We may unify Equations (32) and (33):

$$
I_{i} \delta_{i m}=\frac{V_{m}}{R_{m}}+\sum_{\substack{k=1 \\ k \neq m}}^{N} \frac{V_{m}-V_{k}}{R_{m k}}=V_{m}\left(\frac{1}{R_{m}}+\sum_{\substack{k=1 \\ k \neq m}}^{N} \frac{1}{R_{m k}}\right)-\sum_{\substack{k=1 \\ k \neq m}}^{N} \frac{V_{k}}{R_{m k}} .
$$

so that $m$ is any number between 1 and $N$. In the right side we rearranged the equation, so that the $V_{m}$ terms are put together.

Now dividing by $I_{i}$ and using Equation (29), we have

$$
\delta_{i m}=Z_{m i}\left(\frac{1}{R_{m}}+\sum_{\substack{k=1 \\ k \neq m}}^{N} \frac{1}{R_{m k}}\right)-\sum_{\substack{k=1 \\ k \neq m}}^{N} \frac{Z_{k i}}{R_{m k}}
$$

and we remark that the condition $I_{k}=0$ for $k \neq i$ is satisfied here because we fed only port $i$. One observes that Equations (31) and (35) are the same if we identify:

$$
Y_{m k}=-\frac{1}{R_{m k}} \text { for } \quad(m \neq k)
$$

and

$$
Y_{m m}=\frac{1}{R_{m}}+\sum_{\substack{k=1 \\ k \neq m}}^{N} \frac{1}{R_{m k}}=\frac{1}{R_{m}}-\sum_{\substack{k=1 \\ k \neq m}}^{N} Y_{m k}
$$

where the last equality used Equation (36). So the diagonal element of $\mathcal{Y}$ at position $m$ is the sum of all the conductances connected to port $m$, and the off diagonal element of $\boldsymbol{Y}$ at position $m k$ is minus the conductance between ports $m$ and $k$.

Figure 2 shows the physical interpretation of Equations (36) and (37).

It is worth to remark, that the characteristic admittance matrix $\mathcal{Y}_{0}$ is proportional to the capacitance per length unit matrix $\mathcal{C}$, the way $\mathcal{Z}_{0}$ is proportional to $\mathcal{P}$, see Equation (16). Therefore, the relations above between the admittance matrix and the conductances constituting the network, are the same as the relations between the capacitance matrix $C$ and the capacitances constituting a capacitance network - see (Paul, 1994), Chapter 3.1. Also Figure 2 may be modified to show the calculation of the capacitance matrix, by replacing the resistors by capacitors, and the currents by static charges.

To conclude, if the resistors of a network are known, one may calculate the elements of the $\mathcal{Y}$ matrix using Equations (36) and (37), and invert it to obtain the $\mathcal{Z}$ matrix.

If one needs to implement a network to satisfy a given $\mathcal{Z}$ matrix, say the needed $\mathcal{Z}_{0}$, one inverts $\mathcal{Z}$ to obtain $\mathcal{Y}$, and calculates the resistors between 2 ports (say $m$ and $k$ ), using

$$
R_{m k}=-\frac{1}{Y_{m k}}
$$

and the resistor between port $m$ and common may be extracted from the right side of Equation (37):

$$
R_{m}=\frac{1}{\sum_{k=1}^{N} Y_{m k}}
$$

Two examples of geometries have been calculated in (Ianconescu, 2012) and we show the results of those examples in Figure 3. 


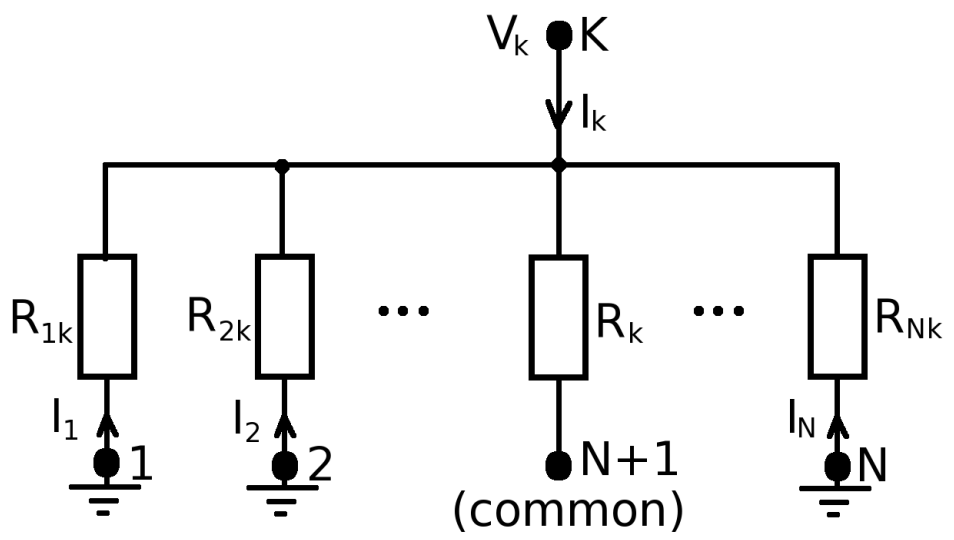

Figure 2. Configuration for calculating $Y_{m k}$ : network fed at conductor $k$ by $V_{k}$, and all other conductors are grounded. The conductors are shown by big points, and the common is by definition at 0 potential. The value of $Y_{m k}$ is given by $I_{m} / V_{k}$. All the resistors not having the index $k$ are at 0 potential on both sides, so the only resistors entering the calculations are $R_{k}$ and $R_{j k}$, for $j \neq k$, and they are all in parallel. The current $I_{m}$ (for $m \neq k$ ) is the current entering from ground into resistor $R_{m k}$, hence equals $-V_{k} / R_{m k}$, and $I_{k}$ is $V_{k}$ divided by the resistors in parallel

The characteristic impedance for the 3 phase geometry in panel (a) of Figure 3 is given by

$$
\mathcal{Z}_{0}=\left(\begin{array}{cc}
2 R / 3 & R / 3 \\
R / 3 & R / 3
\end{array}\right),
$$

where $R=\frac{3}{2} \frac{\eta_{0}}{\pi} \ln \frac{d}{a}, d$ being the side of the triangle and $a$ the radius of the wire in the 3 phase geometry.

The characteristic impedance for the 4 phase geometry in panel (b) of Figure 3 is given by

$$
\mathcal{Z}_{0}=\left(\begin{array}{ccc}
\frac{R_{2}}{4} \frac{R_{2}+3 R_{1}}{R_{2}+R_{1}} & \frac{1}{2} \frac{R_{2} R_{1}}{R_{2}+R_{1}} & \frac{R_{2}}{4} \\
\frac{1}{2} \frac{R_{2} R_{1}}{R_{2}+R_{1}} & \frac{R_{2} R_{1}}{R_{2}+R_{1}} & \frac{1}{2} \frac{R_{2} R_{1}}{R_{2}+R_{1}} \\
\frac{R_{2}}{4} & \frac{1}{2} \frac{R_{2} R_{1}}{R_{2}+R_{1}} & \frac{R_{2}}{4} \frac{R_{2}+3 R_{1}}{R_{2}+R_{1}}
\end{array}\right)
$$

where $R_{2}=\frac{\eta_{0}}{\pi} \ln \frac{d^{2}}{2 a^{2}}$ and $R_{1}=\frac{\eta_{0}}{\pi} \ln \frac{\sqrt{2} d}{a} \ln \frac{d^{2}}{2 a^{2}} / \ln \frac{d}{\sqrt{8} a}, d$ being the side of the rectangle and $a$ the radius of the wire in the 4 phase geometry.

\section{B. The Reflection Matrix}

In case the line is terminated by a loading network having an impedance matrix $\mathcal{Z}_{L} \neq \mathcal{Z}_{0}$, the forward moving wave does not satisfy the termination condition, so there must be a backward wave. Dealing here with the 2 waves we shall denote the forward and backward waves by \pm superscripts, respectively. The most general solution for the voltages vector is a forward wave voltages vector plus a backward wave voltages vector:

$$
\mathbf{V}=\mathbf{V}^{+}+\mathbf{V}^{-}
$$

and keeping the backward current agreed direction toward the positive $z$ axis, same as the forward current has been defined, we have

$$
\mathbf{I}=\mathbf{I}^{+}+\mathbf{I}^{-},
$$

and at the load size we require the termination condition:

$$
\mathbf{V}=\mathcal{Z}_{L} \mathbf{I}
$$

We showed that the connection between forward voltage and current is

$$
\mathbf{V}^{+}=\mathcal{Z}_{0} \mathbf{I}^{+}
$$



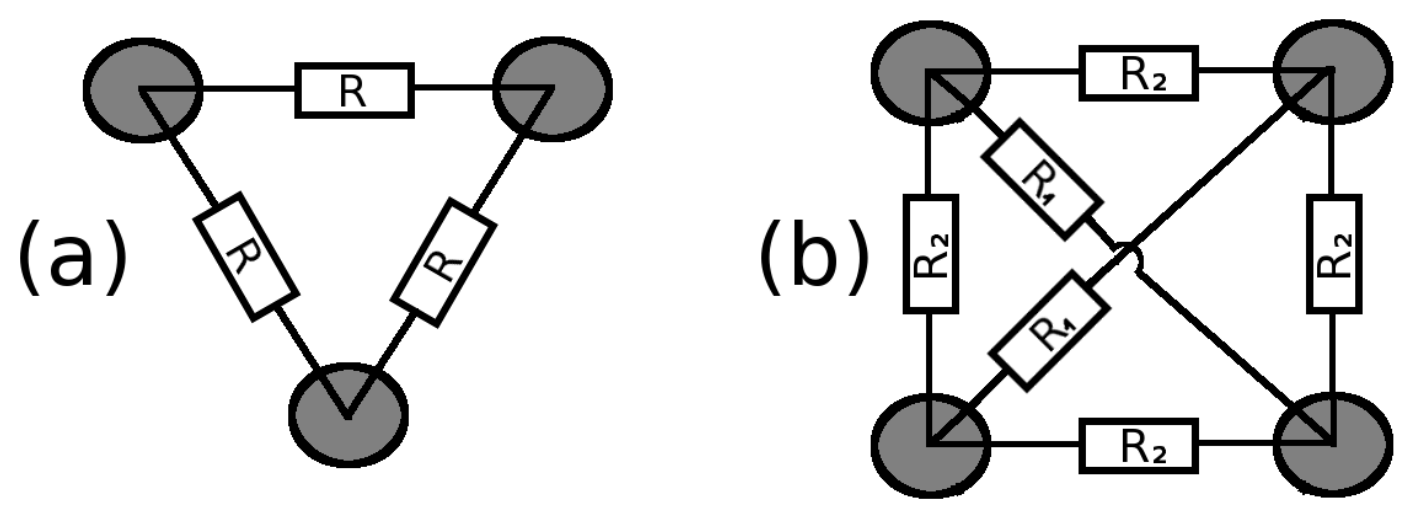

Figure 3. Multiconductor transversal cross sections and their matching networks. Panel (a) shows a 3 phase geometry with wires of radii $a$, at the corners of an equilateral triangle of sides $d$, so that $a \ll d$. The matching network consists of resistors in triangle, where each resistor has the value $R=\frac{3}{2} \frac{\eta_{0}}{\pi} \ln \frac{d}{a}$. Panel (b) shows a 4 phase geometry with wires of radii $a$, at the corners of a square of sides $d$, so that $a \ll d$. The matching network consists of 6 resistors: 4 of value $R_{2}=\frac{\eta_{0}}{\pi} \ln \frac{d^{2}}{2 a^{2}}$ and 2 of value $R_{1}=\frac{\eta_{0}}{\pi} \ln \frac{\sqrt{2} d}{a} \ln \frac{d^{2}}{2 a^{2}} / \ln \frac{d}{\sqrt{8} a}$

(see Equation (15)), hence the connection for the backward wave is

$$
\mathbf{V}^{-}=-\mathcal{Z}_{0} \mathbf{I}^{-}
$$

because $\mathbf{I}^{-}$is defined the direction opposite to its propagation and this may be proved directly by continuing the formalism started in Equation (7) for the backward wave.

By combining Equation (43) with Equations (45) and (46) we obtain

$$
\mathcal{Z}_{0} \mathbf{I}=\mathbf{V}^{+}-\mathbf{V}^{-}
$$

and by adding and subtracting Equations (42) and (47) we obtain:

$$
2 \mathbf{V}^{+}=\mathbf{V}+\mathcal{Z}_{0} \mathbf{I}
$$

and

$$
2 \mathbf{V}^{-}=\mathbf{V}-\mathcal{Z}_{0} \mathbf{I}
$$

We set now the termination condition in Equation (44) into Equations (48) and (49), and by isolating I from Equation (48) and setting it into Equation (49), we obtain the connection between $\mathbf{V}^{-}$and $\mathbf{V}^{+}$:

$$
\mathbf{V}^{-}=\left(\mathcal{Z}_{L}-\mathcal{Z}_{0}\right)\left(\mathcal{Z}_{L}+\mathcal{Z}_{0}\right)^{-1} \mathbf{V}^{+}
$$

which defines the reflection matrix $\Gamma$

$$
\Gamma=\left(\mathcal{Z}_{L}-\mathcal{Z}_{0}\right)\left(\mathcal{Z}_{L}+\mathcal{Z}_{0}\right)^{-1}
$$

in an analogue way to the one dimensional reflection coefficient $\Gamma=\left(Z_{L}-Z_{0}\right) /\left(Z_{L}+Z_{0}\right)$, compare with Wedepohl (1963) and Paul (1994).

One remarks that the reflection matrix is a different concept from the scattering matrix used for a multiport network, although the formula is identical. When calculating the scattering matrix from a passive network, $\mathcal{Z}_{L}$ still represents the network impedance matrix, but $\mathcal{Z}_{0}$ is a diagonal matrix representing the characteristic impedances of the ports.

Here we deal with a single port termination for a multiconductor TEM transmission line and $\mathcal{Z}_{0}$ must not be diagonal.

Because the load impedance matrix may not exist, it would be useful to derive an alternative expression for $\Gamma$ based on the load admittance matrix, yielding

$$
\Gamma=\left(\mathcal{Y}_{0}+\mathcal{Y}_{L}\right)^{-1}\left(\mathcal{Y}_{0}-\mathcal{Y}_{L}\right)
$$


Unlike in the one dimensional case, $\Gamma$ does not need to be the 0 matrix to satisfy $\Gamma \mathbf{V}^{+}=0$. From Equation (52) it is clear that in the case the determinant of $\mathcal{Y}_{0}-\mathcal{Y}_{L}$ is 0 , there exist incident voltage vectors yielding 0 reflection.

\section{Interfacing to the Source Network}

The most general source network has $N(N+1) / 2$ Thevenin branches of source voltage $V_{g}$ is series with a source impedance $R_{g}$ between each pair of conductors. We shall call the resistor and source between conductor $i$ and $N+1$ (the common) $R_{g i}$ and $V_{g i}$ and the resistor and source between conductor $i$ and $j, R_{g i j}$ and $V_{g i j}$, where the + of the source is at the lower index. The naming of the resistors is like in the matching network section.

Calling the current exiting from conductor $i$ of the source network $I_{i}$, and the voltage at conductor $i$ of the source network $V_{i}$ we write the KCL for the intersection $i$

$$
\frac{V_{g i}-V_{i}}{R_{g i}}+\sum_{\substack{j=1 \\ j \neq i}}^{N} \frac{V_{j}+V_{g i j}-V_{i}}{R_{g, i j}}=I_{i}
$$

where $V_{g i j}$ is understood to be taken with positive sign if $i<j$ and vice versa if $i>j$. Equation (53) may be rearranged:

$$
\frac{V_{g i}}{R_{g i}}+\sum_{\substack{j=1 \\ j \neq i}}^{N} \frac{V_{g i j}}{R_{g, i j}}+\sum_{\substack{j=1 \\ j \neq i}}^{N} \frac{V_{j}}{R_{g, i j}}-V_{i}\left(\frac{1}{R_{g i}}+\sum_{\substack{j=1 \\ j \neq i}}^{N} \frac{1}{R_{g, i j}}\right)=I_{i} .
$$

The first 2 expressions represents the sum off all Norton currents connected to conductor $i$, and we shall call it $I_{g} i$. The second 2 expressions may be written as $\left(-\sum_{j=1}^{N} Y_{g i j} V_{j}\right)$, where $Y_{g i j}$ are the elements of the source admittance matrix $\mathcal{Y}_{g}$ - see Equations (36) and (37) defining the admittance matrix elements of a passive network.

Hence we may rewrite Equation (54)

$$
I_{g i}-\sum_{j=1}^{N} Y_{g i j} V_{j}=I_{i}
$$

or in vectorial form

$$
\mathbf{I}_{g}-\boldsymbol{Y}_{g} \mathbf{V}=\mathbf{I} \text {. }
$$

The source network excites a forward wave in the transmission line for which we know the connection between voltage and current $\mathbf{V}=\mathcal{Z}_{0} \mathbf{I}$. So we may isolate the voltage for the forward moving wave

$$
\mathbf{V}=\left(\mathcal{Z}_{0}^{-1}+\mathcal{Y}_{g}\right)^{-1} \mathbf{I}_{g}=\left(\mathcal{Z}_{0}^{-1}+\mathcal{Z}_{g}^{-1}\right)^{-1} \mathbf{I}_{g}
$$

which is a natural extension for the one dimensional case for which we have $I_{g}=V_{g} / Z_{g}$, hence Equation (57) becomes a voltage divider $V=\left(Z_{0}^{-1}+Z_{g}^{-1}\right)^{-1} \frac{V_{g}}{Z_{g}}=V_{g} \frac{Z_{0}}{Z_{0}+Z_{g}}$.

\section{The Differential Representation}

We used so far as dynamical variables the voltages between each conductor and an agreed common conductor, and the currents in each conductor (the common carrying minus the sum of the currents in all other conductors).

The convenience of this representation is the easy way of calculating the magnitudes described in the previous section, but of course this representation is not unique.

Given the voltages vector $\mathbf{V}$, it is sometimes convenient to define the differential voltages vector $\mathbf{V}^{\prime}$, as $V_{1}^{\prime}=V_{1}-V_{2}$, $V_{2}^{\prime}=V_{2}-V_{3}, \ldots V_{N}^{\prime}=V_{N}-0$, which may be written in matrix form

$$
\mathbf{V}^{\prime}=\mathcal{T}_{1} \mathbf{V}
$$

where the matrix $\mathcal{T}_{1}$ has all components 0 , except of the diagonal components which are 1 and the first superdiagonal components which are -1 , and may be expressed as:

$$
T_{1 i, j}=\delta_{i, j}-\delta_{i, j-1},
$$

where $\delta$ is the Kronecker delta, and we assume in this notation that terms with indices smaller than 1 or larger than $N$ are 0 . 
The differential currents $\mathbf{I}^{\prime}$ are loop currents so that we may think of the current $I_{j}$ as the difference between the loop current $I_{j}^{\prime}$ and $I_{j-1}^{\prime}$, so that $I_{1}=I_{1}^{\prime}-0, I_{2}=I_{2}^{\prime}-I_{1}^{\prime}, \ldots I_{N}=I_{N}^{\prime}-I_{N-1}^{\prime}$. This may be written in matrix form

$$
\mathbf{I}=\mathcal{T}_{2} \mathbf{I}^{\prime}
$$

where the matrix $\mathcal{T}_{2}$ has all components 0 , except of the diagonal components which are 1 and the first sub-diagonal components which are -1 , and may be expressed as:

$$
T_{2 i, j}=\delta_{i, j}-\delta_{i, j+1} .
$$

Starting with the conductor to ground representation for which the relation between the forward moving voltage and current vectors is $\mathbf{V}=\mathcal{Z}_{0} \mathbf{I}$, and using Equations (58) and (60), we obtain the differential representation

$$
\mathbf{V}^{\prime}=\mathcal{Z}_{0}^{\prime} \mathbf{I}^{\prime}
$$

where

$$
\mathcal{Z}_{0}^{\prime}=\mathcal{T}_{1} \mathcal{Z}_{0} \mathcal{T}_{2},
$$

and the components of $\mathcal{Z}_{0}^{\prime}$ may be written explicitly in terms of the components of $\mathcal{Z}_{0}$

$$
Z_{0 i, j}^{\prime}=Z_{0 i, j}+Z_{0 i+1, j+1}-Z_{0 i+1, j}-Z_{0 i, j+1} .
$$

again, considering terms with outside range indices as 0 . The usefulness of this transformation is show in the following example.

\section{A. Example: Multiple Parallel Plates}

We will calculate the characteristic impedance matrix for $N+1$ parallel plates at distance $d$ one from the other all having the width $w$, so that $w \gg d$, see Figure 4 .

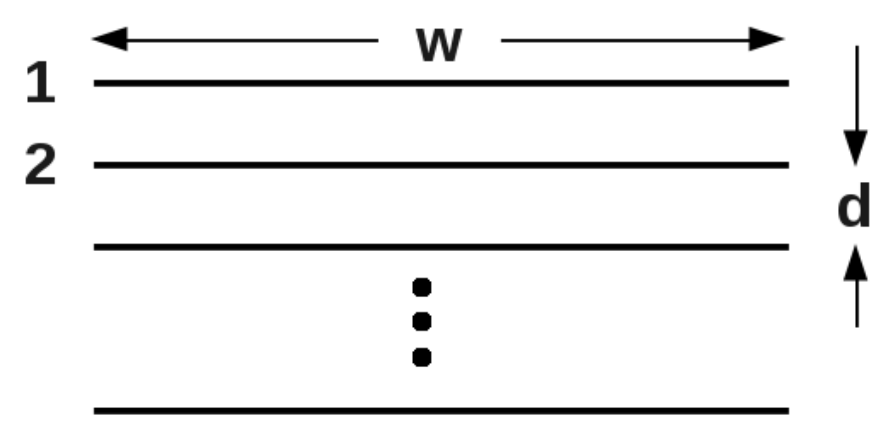

\section{$\mathrm{N}+1$}

Figure 4. Multiconductor waveguide consisting of parallel plates of width $w$, at distance $d$ between them. We assume large plates, i.e. $w \gg d$. The $z$ axis is perpendicular to the paper

To solve Laplace Equation (5) for the transverse potential $V_{\mathrm{tr}}$, we consider plate $j$ to have a surface charge $\eta_{j}$, for $j \neq N+1$, and the charge surface on conductor $N+1$ is $\eta_{N+1}=-\sum_{i=1}^{N} \eta_{i}$. We shall use the expression for the potential due to an infinite plate $\frac{-\eta}{2 \epsilon}|x|$, where $\eta$ is the charge surface and $|x|$ is the distance from the plate. Also we may express the charge per unit length in the $z$ direction as $\lambda=w \eta$. So we may write the potential on conductor $j$ (for $j \neq N+1$ )

$$
\left.V_{\text {tr }}\right|_{\text {cond } j}=\frac{\eta_{j} d}{2 w \epsilon}(N+1-j)+\sum_{i=1}^{j-1} \frac{\eta_{i} d}{2 w \epsilon}(N+1+i-2 j)+\sum_{i=j+1}^{N} \frac{\eta_{i} d}{2 w \epsilon}(N+1-i)
$$

and the potential on conductor $N+1$

$$
\left.V_{\mathrm{tr}}\right|_{\text {cond } N+1}=\sum_{i=1}^{N} \frac{-\eta_{i} d}{2 w \epsilon}(N+1-i)
$$


and express $V_{j}=\left.V_{\mathrm{tr}}\right|_{\text {cond } j}-\left.V_{\mathrm{tr}}\right|_{\text {cond } N+1}$ obtaining

$$
V_{j}=\sum_{i=1}^{j-1} \frac{\eta_{i} d}{w \epsilon}(N+1-j)+\sum_{i=j}^{N} \frac{\eta_{i} d}{w \epsilon}(N+1-i) .
$$

The coefficients are identified as the elastance matrix elements

$$
P_{i j}=\left\{\begin{array}{cc}
\frac{d}{w \epsilon}(N+1-j) & i<j \\
\frac{d}{w \epsilon}(N+1-i) & i \geq j
\end{array},\right.
$$

and the characteristic impedance matrix elements are obtained from Equation (16)

$$
Z_{0 i j}=\left\{\begin{array}{ll}
\frac{\eta d}{w}(N+1-j) & i<j \\
\frac{\eta d}{w}(N+1-i) & i \geq j
\end{array} .\right.
$$

which may also be written as $Z_{0} i j=u_{i-j}(j-i)+N+1-j$, where $u$ is the discreet step function, i.e. equals 1 for non negative index and zero otherwise.

This form does not emphasize the separability of this configuration, so we would like to transform to the differential representation with Equation (64). After some algebra, noting that $u_{i-j}-u_{i-j-1}=\delta_{i, j}$, one obtains

$$
Z_{0 i j}^{\prime}=\delta_{i, j} \frac{\eta d}{w}
$$

or in matrix form $\mathcal{Z}_{0}^{\prime}=\mathcal{I} \frac{\eta d}{w}, \mathcal{I}$ being the unit matrix. So the differential representation results in $N$ separate TEM waveguides, each having the characteristic impedance $\frac{\eta d}{w}$.

This was a special example of separable configuration, but the differential representation may be useful also for non separable configurations, because it emphasizes better the "local impedance", i.e. the connection between the voltage difference between 2 adjacent conductors, and the loop current between them, as we shall see in the next section.

\section{Flat Cable Analysis}

The flat cable is the most common multiconductor transmission line, hence we dedicate this section to flat cables and we shall calculate here the characteristic impedance matrix for a flat cable.

The distance between the wires (called pitch) is $d$ and the conductor radius is $a$, and we consider the common case of $a \ll d$ - see Figure 5 .

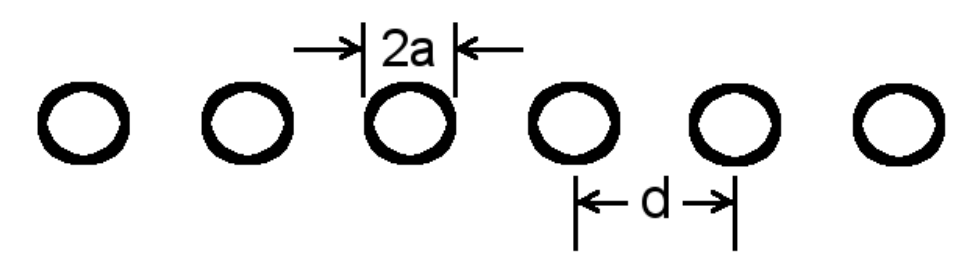

Figure 5. The geometry of a flat cable: equidistant conductors of radii $a$. The distance between the conductors $d$ is called pitch and is typically much bigger than the conductors radius. We consider the surrounding media to be homogeneous

The wires are numbered $1,2, . . N, N+1$. We assign the charges per length unit $\lambda_{1}, \lambda_{2}, . . \lambda_{N}$, and the $N+1$ wire (considered the common) has the charges per length unit

$$
\lambda_{N+1}=-\sum_{i=1}^{N} \lambda_{i}
$$


The solution of the Laplace equation, i.e. the potential at any location may be expressed using the thin wire approximation

$$
V_{\mathrm{tr}}=-\sum_{i=1}^{N+1} \frac{\lambda_{i}}{2 \pi \epsilon} \ln r_{i}
$$

where $\epsilon$ is some equivalent dielectric constant, i.e. $\epsilon=\epsilon_{0} \epsilon_{r}, \epsilon_{r}$ is usually between 1.5 and 3, and $r_{j}$ is the distance from conductor $j$.

By putting Equation (71) into Equation (72), we get

$$
V_{\mathrm{tr}}=\sum_{i=1}^{N} \frac{\lambda_{i}}{2 \pi \epsilon} \ln \frac{r_{N+1}}{r_{i}}
$$

and to express the potential on the conductor $j$ (for $j \neq N+1$ ) we set $r_{N+1}=d\left(N+1-j\right.$ ). Also, we set $r_{i}=d|j-i|$ if $i \neq j$ and $r_{i}=a$ if $i=j$, according to the thin wire approximation, obtaining

$$
\left.V_{\text {tr }}\right|_{\text {conductor } \mathrm{j}}=\frac{\lambda_{j}}{2 \pi \epsilon} \ln \frac{d(N+1-j)}{a}+\sum_{\substack{i=1 \\ i \neq j}}^{N} \frac{\lambda_{i}}{2 \pi \epsilon} \ln \frac{N+1-j}{|j-i|},
$$

and on conductor $N+1$, we set $r_{N+1}=a$ and $r_{i}=d(N+1-i)$

$$
\left.V_{\text {tr }}\right|_{\text {conductor } \mathrm{N}+1}=\sum_{i=1}^{N} \frac{\lambda_{i}}{2 \pi \epsilon} \ln \frac{a}{d(N+1-i)} .
$$

The potential difference $V_{j}$ is given by $\left.V_{\text {tr }}\right|_{\text {conductor } \mathrm{j}}-\left.V_{\text {tr }}\right|_{\text {conductor } \mathrm{N}+1}$ and comes out:

$$
V_{j}=\frac{\lambda_{j}}{\pi \epsilon} \ln \frac{d(N+1-j)}{a}+\sum_{\substack{i=1 \\ i \neq j}}^{N} \frac{\lambda_{i}}{2 \pi \epsilon} \ln \frac{d(N+1-j)(N+1-i)}{a|j-i|},
$$

and this defines the elements of the elastance matrix

$$
P_{i j}=\left\{\begin{array}{ll}
\frac{1}{\pi \epsilon} \ln \frac{d(N+1-j)}{a} & i=j \\
\frac{1}{2 \pi \epsilon} \ln \frac{d(N+1-j)(N+1-i)}{a|j-i|} & i \neq j
\end{array},\right.
$$

and the characteristic impedance matrix elements are obtained from Equation (16)

$$
Z_{0 i j}=\left\{\begin{array}{ll}
\frac{\eta}{\pi} \ln \frac{d(N+1-j)}{a} & i=j \\
\frac{\eta}{2 \pi} \ln \frac{d(N+1-j)(N+1-i)}{a|j-i|} & i \neq j
\end{array},\right.
$$

where $\eta=\sqrt{\epsilon / \mu_{0}}$.

It will be convenient to see also the differential representation of the characteristic impedance matrix. Using Equation (64) we obtain:

$$
Z_{0 i j}^{\prime}=\left\{\begin{array}{ll}
\frac{\eta}{\pi} \ln \frac{d}{a} & i=j \\
\frac{-\eta}{2 \pi} \ln \frac{d}{2 a} & |i-j|=1 \\
\frac{\eta}{2 \pi} \ln \left(1-\frac{1}{|j-i|^{2}}\right) & |i-j|>1
\end{array},\right.
$$

which is much simpler than regular representation.

We will calculate the balanced crosstalk on a 9L280XX Belden flat cable. The datasheet of the cable can be found at (Belden flat cables datasheet, n.d.)). The cables have a pitch of $d=0.05 "=1.27 \mathrm{~mm}$ and wires of type 28 AWG, having a radius of $a=0.1605 \mathrm{~mm}$. The measurement configuration is shown in Figure 6.

We shall first suppose the far end is matched and than show how to match it. So given the far end is matched, we have one single forward voltages vector wave $\mathbf{V}$ which satisfies the equation $\mathbf{V}=\mathcal{Z}_{0} \mathbf{I}$, I being the forward currents 


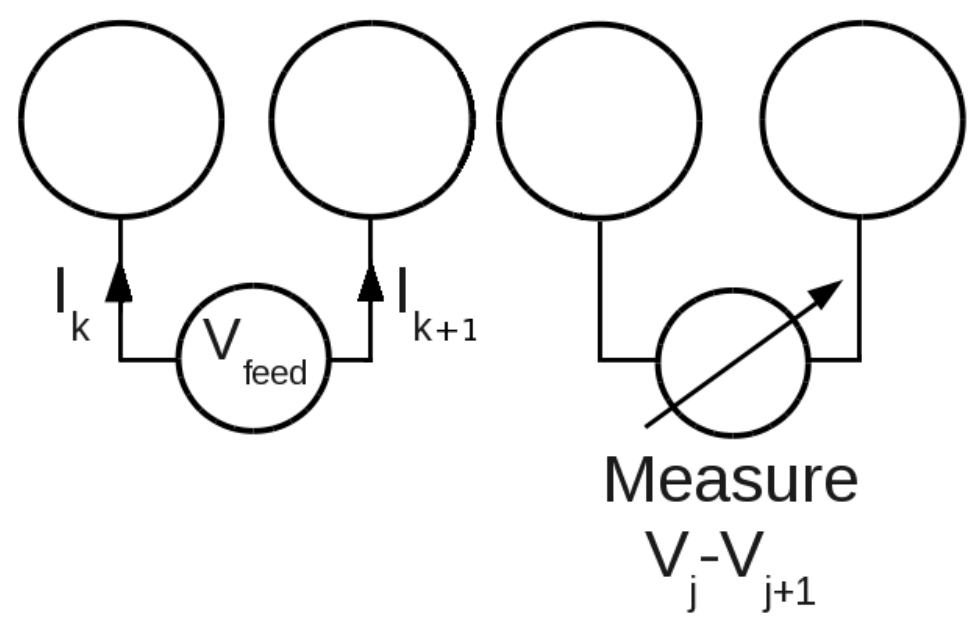

Figure 6. A balanced cross talk measurement on a flat cable. The conductors $k$ and $k+1$ are differentially fed, and are called the drive pair, and one measures the voltage between two other conductors $j$ and $j+1$, called the sample pair. The biggest crosstalk effect is on neighboring conductors, so we take $j=k+2$. The far end is matched

vector. But all the components of the currents vector are 0 except of the $k$ and $k+1$ components. Also we know that $I_{k+1}=-I_{k}$, so we may write the $n$ component of the forward voltage vector:

$$
V_{n}=\left(Z_{0 n, k}-Z_{0 n, k+1}\right) I_{k}
$$

It looks quite natural that having a non zero current only on conductors $k$ and $k+1$, one would need at the far end to connect a resistance only between those conductors, so let us call this resistance $R_{0}$. According to Equations (36) and (37), connecting only this resistor defines the components of the load admittance matrix

$$
Y_{L m, n}=\frac{1}{R_{0}}\left(\delta_{m, k} \delta_{n, k}+\delta_{m, k+1} \delta_{n, k+1}-\delta_{m, k} \delta_{n, k+1}-\delta_{m, k+1} \delta_{n, k}\right),
$$

so $\mathcal{Y}_{L}$ has only 4 non zero components, 2 diagonal components of value $1 / R_{0}$ and 2 off diagonal components of value $-1 / R_{0}$.

For having a 0 reflected voltage one needs $\left(\mathcal{Y}_{0}-\mathcal{Y}_{L}\right) \mathbf{V}=0$ (see Equation (52) and comments after it). Hence we calculate first the $m$ component of $\mathcal{Y}_{0} \mathbf{V}$, so using $V_{n}$ from Equation (80) we obtain

$$
\left[\mathcal{Y}_{0} \mathbf{V}\right]_{m}=\sum_{n} Y_{0 m, n} V_{n}=I_{k}\left(\delta_{m, k}-\delta_{m, k+1}\right),
$$

because $\mathcal{Y}_{0}$ is the inverse matrix of $\mathcal{Z}_{0}$. Now the $m$ component of $\mathcal{Y}_{L} \mathbf{V}$ yields

$$
\left[\mathcal{Y}_{L} \mathbf{V}\right]_{m}=\sum_{n} Y_{L m, n} V_{n}=\frac{I_{k}}{R_{0}}\left(\delta_{m, k}-\delta_{m, k+1}\right)\left(Z_{0 k, k}-2 Z_{0 k+1, k}+Z_{0 k+1, k+1}\right),
$$

and we see that the value of $R_{0}$ needed to make the results of Equations (82) and (83) identical is

$$
R_{0}=Z_{0 k, k}-2 Z_{0 k+1, k}+Z_{0 k+1, k+1}=Z_{0 k, k}^{\prime}=\frac{\eta}{\pi} \ln (d / a),
$$

where for the last expression we used the relation (64) and Equation (79), obtaining the diagonal element of the characteristic impedance matrix in the differential representation.

Also, one remarks that the value of $R_{0}$ should be the equivalent of what Belden (Belden flat cables datasheet, n.d.) call in their datasheet the "impedance".

The value they give is $105 \Omega$ and for comparing we need to know the value of $\eta=377 \Omega / \sqrt{\epsilon_{r}}$, where $\epsilon_{r}$ is some equivalent relative dielectric coefficient which is influenced by the isolation material and the surrounding air. 
The value of $\epsilon_{r}$ may be calculated using the propagation delay from the datasheet, given as $4.6 \mathrm{nsec} / \mathrm{m}$, so that $\sqrt{\epsilon_{r}}$ is the light velocity in vacuum, multiplied by the propagation delay, resulting in 1.38 . Using the above, $R_{0}$ comes out $180 \Omega$, which is about 1.7 times higher than the $105 \Omega$ in the datasheet. I suppose the reason for this discrepancy are very big losses given in the datasheet, which imply dispersion and do no allow a simple time domain analysis like this one.

In spite of the discrepancy in the characteristic impedance value, the calculated cross talk compares well with the datasheet, because this value depends on a relation between the characteristic impedance matrix elements, as we shall see below.

So the feeding voltage is expressed as $V_{\text {feed }}=V_{k}-V_{k+1}$ and using Equation (80) we obtain

$$
V_{\text {feed }}=\left(Z_{0 k, k}-2 Z_{0 k+1, k}+Z_{0 k+1, k+1}\right) I_{k}=I_{k} R_{0}
$$

Similarly, the sample voltage is $V_{\text {sample }}=V_{k+2}-V_{k+3}$ and using Equation (80) we obtain $V_{\text {sample }}=\left(Z_{0 k+2, k}-\right.$ $\left.Z_{0 k+2, k+1}+Z_{0 k+3, k+1}-Z_{0 k+3, k}\right) I_{k}$. By comparing this with Equation (64), we see that $V_{\text {sample }}=Z_{0 k+2, k}^{\prime} I_{k}$ and from Equation (79) we obtain

$$
V_{\text {sample }}=I_{k} \frac{\eta}{2 \pi} \ln (3 / 4)
$$

The crosstalk is defined as the absolute value of the relation between $V_{\text {sample }}$ and $V_{\text {feed }}$ :

$$
\text { Crosstalk }=\left|\frac{V_{\text {sample }}}{V_{\text {feed }}}\right|=\frac{\ln (4 / 3)}{2 \ln (d / a)}=0.06954=-23.1 \mathrm{~dB}
$$

This value is also called crosstalk isolation and is given in the Belden datasheet (Belden flat cables datasheet, n.d.) as $-20 \mathrm{~dB}$, showing that the above calculation fits well the measurement.

\section{Multiconductor TEM in Steady State Harmonic Excitation}

In this section we generalize the multiconductor TEM for the case of steady state harmonic excitation. One may rewrite the one dimensional wave Equation (6) by replacing $\partial_{t}$ by $j \omega$, or alternatively replace the solution function (say in Equation (7)) $f(p)=\exp (j \omega p)$, to obtain the phasor solutions of the form $\exp (\mp j \beta z)$ for the forward and backward moving waves, respectively, where

$$
\beta \equiv \omega / v
$$

is the wave number. For harmonic steady state we need to develop formulas to move along transmission line and express quantities at some location $z$, given that they are known at other location say $z_{1}$.

The basic solutions for the voltage, i.e. the forward an backward moving voltages behave according to

$$
\mathbf{V}^{ \pm}(z)=\mathbf{V}^{ \pm}\left(z_{1}\right) e^{\mp j \beta\left(z-z_{1}\right)},
$$

and the forward an backward moving currents behave the same

$$
\mathbf{I}^{ \pm}(z)=\mathbf{I}^{ \pm}\left(z_{1}\right) e^{\mp j \beta\left(z-z_{1}\right)},
$$

and the connection between forward/backward voltages and currents is the same like for any excitation

$$
\mathbf{V}^{ \pm}(z)= \pm \mathcal{Z}_{0} \mathbf{I}^{ \pm}(z) \text {. }
$$

we define the reflection matrix at any location $z$ by the connection between the backward and forward voltages

$$
\mathbf{V}^{-}(z)=\Gamma(z) \mathbf{V}^{+}(z) .
$$

where $\Gamma$ is now a complex phasor matrix. By setting Equation (89) into Equation (92) and by replacing $\mathbf{V}^{-}\left(z_{1}\right)=$ $\Gamma\left(z_{1}\right) \mathbf{V}^{+}\left(z_{1}\right)$ we obtain:

$$
\Gamma(z) \mathbf{V}^{+}\left(z_{1}\right)=e^{2 j \beta\left(z-z_{1}\right)} \Gamma\left(z_{1}\right) \mathbf{V}^{+}\left(z_{1}\right),
$$

which must hold for any set of voltages $\mathbf{V}^{+}\left(z_{1}\right)$, hence we are left with

$$
\Gamma(z)=e^{2 j \beta\left(z-z_{1}\right)} \Gamma\left(z_{1}\right),
$$

which is the generalization of the one dimensional case $\Gamma(z)=e^{2 j \beta\left(z-z_{1}\right)} \Gamma\left(z_{1}\right)$. 
The Equations (42) and (47) hold for harmonic steady state at any $z$, so we write them at location $z$ and use Equation (89):

$$
\begin{gathered}
\mathbf{V}(z)=\mathbf{V}^{+}(z)+\mathbf{V}^{-}(z)=\mathbf{V}^{+}\left(z_{1}\right) e^{-j \beta\left(z-z_{1}\right)}+\mathbf{V}^{-}\left(z_{1}\right) e^{j \beta\left(z-z_{1}\right)} \\
\mathcal{Z}_{0} \mathbf{I}(z)=\mathbf{V}^{+}(z)-\mathbf{V}^{-}(z)=\mathbf{V}^{+}\left(z_{1}\right) e^{-j \beta\left(z-z_{1}\right)}-\mathbf{V}^{-}\left(z_{1}\right) e^{j \beta\left(z-z_{1}\right)},
\end{gathered}
$$

Now we use Equations (48) and (49) at location $z_{1}$ to extract $\mathbf{V}^{+}\left(z_{1}\right)$ and $\mathbf{V}^{-}\left(z_{1}\right)$ and set into Equations (95) and (96):

$$
\mathbf{V}(z)=\frac{1}{2}\left[\mathbf{V}\left(z_{1}\right)+\mathcal{Z}_{0} \mathbf{I}\left(z_{1}\right)\right] e^{-j \beta\left(z-z_{1}\right)}+\frac{1}{2}\left[\mathbf{V}\left(z_{1}\right)-\mathcal{Z}_{0} \mathbf{I}\left(z_{1}\right)\right] e^{j \beta\left(z-z_{1}\right)}
$$

and after rearranging

$$
\mathbf{V}(z)=\mathbf{V}\left(z_{1}\right) \cos \beta\left(z-z_{1}\right)-j \mathcal{Z}_{0} \mathbf{I}\left(z_{1}\right) \sin \beta\left(z-z_{1}\right)
$$

and similarly for the current

$$
\mathbf{I}(z)=\mathbf{I}\left(z_{1}\right) \cos \beta\left(z-z_{1}\right)-j \mathcal{Y}_{0} \mathbf{V}\left(z_{1}\right) \sin \beta\left(z-z_{1}\right) .
$$

We define here the local impedance matrix as:

$$
\mathbf{V}(z)=\mathcal{Z}(z) \mathbf{I}(z),
$$

so by replacing $\mathbf{V}\left(z_{1}\right)=\mathcal{Z}\left(z_{1}\right) \mathbf{I}\left(z_{1}\right)$ in Equations (98) and (99), one obtains

$$
\mathbf{V}(z)=\left[\mathcal{Z}\left(z_{1}\right) \cos \beta\left(z-z_{1}\right)-j \mathcal{Z}_{0} \sin \beta\left(z-z_{1}\right)\right] \mathbf{I}\left(z_{1}\right)
$$

and

$$
\mathbf{I}(z)=\left[\mathcal{I} \cos \beta\left(z-z_{1}\right)-j \boldsymbol{y}_{0} \mathcal{Z}\left(z_{1}\right) \sin \beta\left(z-z_{1}\right)\right] \mathbf{I}\left(z_{1}\right),
$$

where $\mathcal{I}$ is the unit matrix. Now isolating $\mathbf{I}\left(z_{1}\right)$ from Equation (102), setting it in Equation (101) and taking $\cos \beta\left(z-z_{1}\right)$ outside parenthesis yields

$$
\mathbf{V}(z)=\left[\mathcal{Z}\left(z_{1}\right)-\mathcal{Z}_{0} \tan \beta\left(z-z_{1}\right)\right]\left[\mathcal{I}-j \mathcal{Y}_{0} \mathcal{Z}\left(z_{1}\right) \tan \beta\left(z-z_{1}\right)\right]^{-1} \mathbf{I}(z),
$$

from which we get

$$
\mathcal{Z}(z)=\left[\mathcal{Z}\left(z_{1}\right)-j \mathcal{Z}_{0} \tan \beta\left(z-z_{1}\right)\right]\left[\mathcal{I}-j \mathcal{Y}_{0} \mathcal{Z}\left(z_{1}\right) \tan \beta\left(z-z_{1}\right)\right]^{-1},
$$

which may be rearranged to

$$
\mathcal{Z}(z)=\left[\mathcal{Z}\left(z_{1}\right)-j \mathcal{Z}_{0} \tan \beta\left(z-z_{1}\right)\right]\left[\mathcal{Z}_{0}-j \mathcal{Z}\left(z_{1}\right) \tan \beta\left(z-z_{1}\right)\right]^{-1} \mathcal{Z}_{0}
$$

which is the analogue of the one dimensional formula $Z(z)=Z_{0} \frac{Z\left(z_{1}\right)-j Z_{0} \tan \beta\left(z-z_{1}\right)}{Z_{0}-j Z\left(z_{1}\right) \tan \beta\left(z-z_{1}\right)}$.

For the case the matrix $\mathcal{Z}\left(z_{1}\right)$ does not exist it is useful to express the above connection via admittances. Some matrix algebra yields

$$
\boldsymbol{Y}(z)=\left[\mathcal{Y}\left(z_{1}\right)-j \mathcal{Y}_{0} \tan \beta\left(z-z_{1}\right)\right]\left[\mathcal{Y}_{0}-j \mathcal{Y}\left(z_{1}\right) \tan \beta\left(z-z_{1}\right)\right]^{-1} \mathcal{Y}_{0},
$$

and the connection between $\Gamma(z), \mathcal{Z}(z)$ and $\mathcal{Y}(z)$ is given by

$$
\Gamma(z)=\left(\mathcal{Z}(z)-\mathcal{Z}_{0}\right)\left(\mathcal{Z}(z)+\mathcal{Z}_{0}\right)^{-1}=\left(\mathcal{Y}_{0}+\mathcal{Y}(z)\right)^{-1}\left(\boldsymbol{Y}_{0}-\mathcal{Y}(z)\right)
$$

The solution for an harmonic steady state multiconductor transmission line of length $l$, given the sources and the load impedance matrix is similar to the one dimensional case. First we set

$$
\mathcal{Z}(l)=\mathcal{Z}_{L}
$$

where $\mathcal{Z}_{L}$ is the load impedance matrix. Then we use Equation (105) to calculate the impedance matrix at the source location $\left(z=0\right.$ and $\left.z_{1}=l\right)$

$$
\mathcal{Z}_{\text {in }}=\mathcal{Z}(0)=\left[\mathcal{Z}(l)+j \mathcal{Z}_{0} \tan \beta l\right]\left[\mathcal{Z}_{0}+j \mathcal{Z}(l) \tan \beta l\right]^{-1} \mathcal{Z}_{0}
$$


and this is the input impedance matrix of the multiconductor transmission line. Next we use the interface to source formula Equation (57), developed for transients, only $\mathcal{Z}_{0}$ has to be replaced by $\mathcal{Z}_{\text {in }}$, to calculate the voltage near the source

$$
\mathbf{V}(0)=\left(\mathcal{Z}_{\text {in }}^{-1}+\mathcal{Z}_{g}^{-1}\right)^{-1} \mathbf{I}_{g}
$$

and the current near the source is

$$
\mathbf{I}(0)=\mathcal{Z}_{\text {in }}^{-1} \mathbf{V}(0)=\left(\mathcal{I}+\mathcal{Z}_{g}^{-1} \mathcal{Z}_{\text {in }}\right)^{-1} \mathbf{I}_{g}
$$

Knowing $\mathbf{V}(0)$ and $\mathbf{I}(0)$, one may calculate $\mathbf{V}(z)$ and $\mathbf{I}(z)$ for any location $z$ using Equations (98) and (99) (by setting $\left.z_{1}=0\right)$.

The power of a forward moving wave (see Equation (28)) is also easily generalized for the time harmonic case

$$
P^{+}=\mathbf{V}^{+T} \mathcal{Z}_{0}^{-1} \mathbf{V}^{+*}
$$

where the $*$ denotes the complex conjugate, and according to Equation (89), the result is $z$ independent and real. Also, defining the magnitudes of the phasors we use as RMS values, we do not need a 1/2 factor in Equation (112).

It would be interesting to check what is the multidimensional analog to the standing wave ratio (SWR). As explained in Section 5.B, the reflection at the termination may depend on the incident voltages vector itself, therefore it is impossible in the general case to generalize the SWR (in (Paul, 1994) the SWR is considered only for the one dimensional case). Another way of understanding this is to look at a separable configuration - see Figure 4. For this case one finds that $\mathcal{Z}_{0}$ is diagonal in the differential representation (see Equation (70)), and we have $N$ separate waveguides, each one of them may have its own SWR.

But there is a special case of interest: if the load impedance matrix $\mathcal{Z}_{L}$, the characteristic impedance matrix $\mathcal{Z}_{0}$ and the source impedance matrix $\mathcal{Z}_{g}$ are all scalar multiples of the same matrix $Q$, one may generalize the SWR and work with scalar waves.

In this case we have $Z_{L}=Q Z_{L}, Z_{0}=Q Z_{0}$ and $Z_{g}=Q Z_{g}$ where $Z_{L}, Z_{0}$ and $Z_{g}$ are impedances. For this case Equation (105) may be written as

$$
\mathcal{Z}(z)=Q Z_{0} \frac{Z\left(z_{1}\right)-j Z_{0} \tan \beta\left(z-z_{1}\right)}{Z_{0}-j Z\left(z_{1}\right) \tan \beta\left(z-z_{1}\right)} \equiv Q Z(z)
$$

and Equation (107) as

$$
\Gamma(z)=I \frac{Z(z)-Z_{0}}{Z(z)+Z_{0}}
$$

also the input impedance matrix entering Equation (110) may be written as $Z_{\text {in }}=Q Z_{\text {in }}$, so that Equation (110) becomes

$$
\mathbf{V}(0)=\frac{Z_{\text {in }} Z_{g}}{Z_{\text {in }}+Z_{g}} Q \mathbf{I}_{g},
$$

and the power of a forward moving wave becomes

$$
P^{+}=\frac{1}{Z_{0}} \mathbf{V}^{+T} Q^{-1} \mathbf{V}^{+*}
$$

\section{A. Example: Three Phase Network}

We shall use here the cross section geometry of 3 thin wires of radii $a$ on the edges of an equilateral triangle of side $d$, so that $d \gg a$, see Figure 3, panel (a).

The characteristic impedance matrix for this geometry is given in Equation (40), and may be rewritten as

$$
Z_{0}=Z_{0} Q
$$

where $Q$ is the following matrix

$$
Q=\left(\begin{array}{ll}
2 / 3 & 1 / 3 \\
1 / 3 & 2 / 3
\end{array}\right)
$$

and

$$
Z_{0}=\frac{3 \eta_{0}}{2 \pi} \ln (d / a)
$$




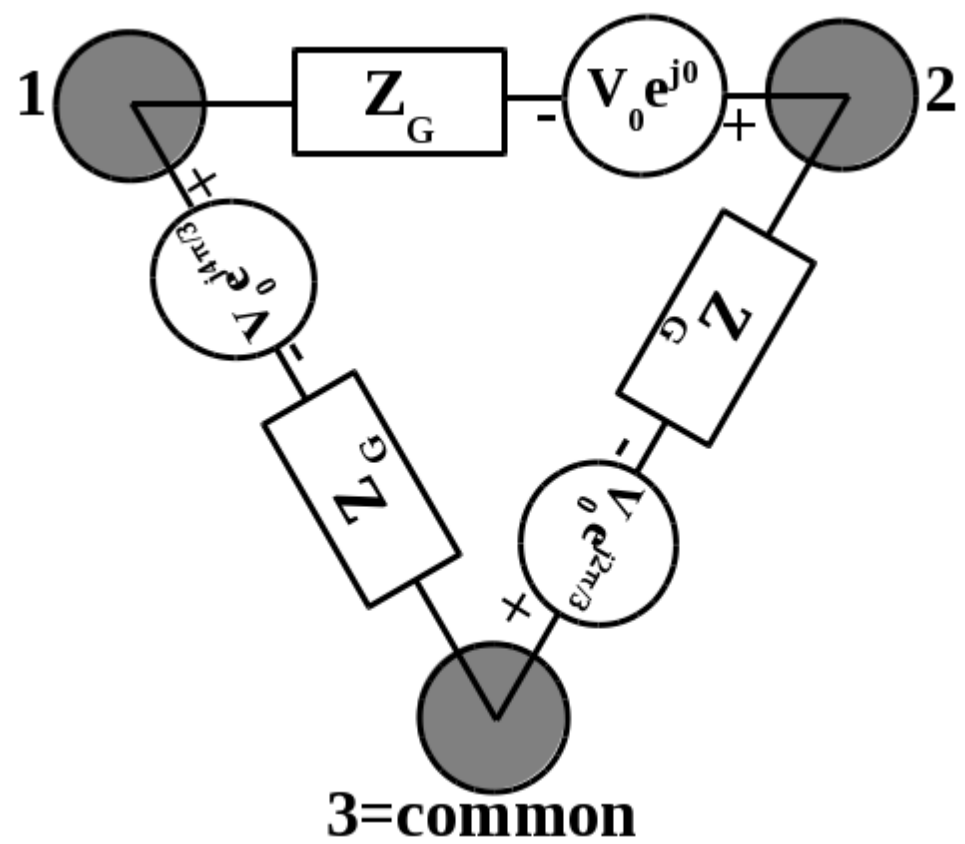

Figure 7. Three phase generator with internal impedance $Z_{g}$ feeding the 3 phase symmetric geometry transmission line with characteristic impedance matrix given in Equation (117). The magnitude of the voltage is $V_{0}$ RMS

has the value of the matching resistors in Figure 3 panel (a).

The network is fed by a 3 phase generator, each phase having the generator impedance $Z_{g}$, see Figure 7 .

The purpose of this example is to show the power transfer, so to simplify it, we shall consider the 3 phase transmission line matched, hence $Z_{\text {in }}=Z_{0}$. We first obtain the Norton currents vector $\mathbf{I}_{g}$ (see Equation (54))

$$
\mathbf{I}_{g}=\frac{V_{0}}{Z_{g}}\left(\begin{array}{c}
\exp (j 4 \pi / 3)-1 \\
1-\exp (j 2 \pi / 3)
\end{array}\right)=\frac{\sqrt{3} V_{0}}{Z_{g}}\left(\begin{array}{c}
\exp (j 5 \pi / 6) \\
\exp (j \pi / 6)
\end{array}\right),
$$

and using Equation (115) we obtain the voltage vector at the beginning of the line

$$
\mathbf{V}(0)=\frac{\sqrt{3} V_{0} Z_{0}}{Z_{0}+Z_{g}} Q\left(\begin{array}{l}
\exp (j 5 \pi / 6) \\
\exp (j \pi / 6)
\end{array}\right),
$$

Being matched, this voltage vector is the forward moving voltage vector which only changes its phase in propagation. Using Equation (116), we obtain the power

$$
P=\frac{3}{Z_{0}}\left|\frac{V_{0} Z_{0}}{Z_{0}+Z_{g}}\right|^{2}(\exp (j 5 \pi / 6) \exp (j \pi / 6)) Q\left(\begin{array}{l}
\exp (j 5 \pi / 6) \\
\exp (j \pi / 6)
\end{array}\right)^{*},
$$

The last matrices multiplication results in 1 , and we identify $V_{0} Z_{0} /\left(Z_{0}+Z_{g}\right)$ as the voltage on each phase $V_{\mathrm{ph}}$ of the matched load $Z_{\mathrm{ph}}=Z_{0}$, resulting in the usual 3 phase power $3\left|V_{\mathrm{ph}}\right|^{2} / Z_{\mathrm{ph}}$.

Of course, this result is valid for the same load we considered above, on any geometry of 3 phase transmission line, provided the length is short compared to the wavelength, so that the characteristic impedance does not count when reflecting the load toward the generator.

\section{Conclusions}

We developed here a different derivation for TEM waves guided by many perfect conductors in a homogeneous media, the connection between the multiconductor transmission line, source and load, and formulated the power transfer.

Using this formalism, we calculated the cross talk for a flat cable, and our result compares well with the datasheet. It is to be mentioned that any multiconductor transmission line which does not have a diagonal representation suffers from cross talk. 
The crosstalk is a major problem, due to which one has to reduce the information rate, therefore it deserves a special attention. An additional work on avoiding crosstalk is in preparation.

We generalized the formulation for time harmonic, and showed that in the general case the SWR is not defined, unless special symmetry conditions are satisfied, and for such case, one may express the propagation problem in a scalar form. Using this scalar form, we calculated the power transmitted into a 3 phase transmission line, obtaining the known expression for the power in 3 phase transmission lines.

\section{References}

Belden flat cables datasheet. (n.d.). Retrieved from http://www.belden.com/resourcecenter/tools/flat-cable.cfm

Chen, B., \& Lu, X. (2011). Reflection and Propagation of Electromagnetic Wave in Time-varying Medium. MAS, $5(4), 243-252$.

Ianconescu, R. (2012, Nov). Polyphase guided TEM waves. Presented at IEEE 27-th Convention of Electrical and Electronics Engineers in Israel, Eilat, November 14-17. http://dx.doi.org/10.1109/EEEI.2012.6377001

Galloway, R. H., Shorrocks, W. B., \& Wedepohl, L. M. (1964, Dec). Calculation of electrical parameters for short and long polyphase transmission lines. PROC. IEE, 111(12), 2051-2059. http://dx.doi.org/10.1049/piee.1964.0331

Olyslager, F., De Zutter, D., \& de Hoop, A. T. (1994). New Reciprocal Circuit Model for Lossy Waveguide Structures Based on the Orthogonality of the Eigenmodes. IEEE Trans. Microw. Theory Techn., 42(12), 2261-2269.http://dx.doi.org/10.1109/22.339751

Olyslager, F. (1999). Electromagnetic Waveguides and Transmission Lines. OUP Oxford.

Orfanidis, S. J. (2002). Electromagnetic Waves and Antennas. Rutgers University.

Park, R. H. (1929, July). Two-reaction theory of synchronous machines generalized method of analysis-part I. Transactions of the American Institute of Electrical Engineers, 48(3), 716-727. http://dx.doi.org/10.1109/T-AIEE.1929.5055275

Park, R. H. (1933, June). Two-reaction theory of synchronous machines-II. Transactions of the American Institute of Electrical Engineers, 52(2), 352-354. http://dx.doi.org/10.1109/T-AIEE.1933.5056309

Paul, C. R. (1994). Analysis of Multiconductor Transmission Lines. New York: Wiley.

Pozar, D. M. (2009). Microwave Engineering. Wiley India Pvt.

Staelin, D. H., Morgenthaler, A., \& Kong, J. A. (1994). Electromagnetic Waves. Prentice Hall.

Wedepohl, L. M. (1963, Dec). Application of matrix methods to the solution of traveling-wave phenomena in polyphase systems. PROC. IEE, 110(12), 2200-2212. http://dx.doi.org/10.1049/piee.1963.0314

\section{Copyrights}

Copyright for this article is retained by the author(s), with first publication rights granted to the journal.

This is an open-access article distributed under the terms and conditions of the Creative Commons Attribution license (http://creativecommons.org/licenses/by/3.0/). 